\title{
Corporate Governance and Firm Performance in India: An Empirical Research
}

\author{
T.Sita Ramaiah, N.Akbar Jan, Radha Mohan Chebolu, Thangaraja Arumugam, A.K. \\ Subramani
}

\begin{abstract}
The point of the exploration is to look at the relationship among corporate administration and firm execution. In this examination study board information of $S \& P$ BSE-100 listed organizations from 2011-2018 and LSDV board information model, 2SLS model are utilized as instruments for investigation of the information. Where Market to Book worth and Tobin $Q$ are taken as the needy variable while size of the board, freedom with the board; compensation given for the board, advertiser shareholding are considered as autonomous factors. The result of the examination uncovers that great corporate administration practices actualized by organizations are decidedly identified with money related execution. The finding of the examination uncovers that littler sheets are probably going to be progressively proficient in observing execution (Fuerst and Kang 2000; Loderer and Peyer 2002). The present examination affirms that there is a negative relationship among board freedom and execution of the firm and higher level of outside executives negatively affect firm execution (Yermack, 1996; Klein, 1998).
\end{abstract}

Keywords: Corporate Governance, Firm Performance, Board Independence, Panel data analysis

\section{INTRODUCTION}

The key principle of a decent corporate administration structure is to expand the commitment of the firm to the whole economy, i.e., which includes all partners. With this definition, corporate administration describes about the relationship among investors, loan bosses, enterprises, budgetary markets, foundations and workers (Classens, 2006). Hereafter it is engaged that great corporate administration expands the consistency and execution of the firm by progressing in the direction of the practical financial advancement (Mallin, 2008). Great corporate administration makes ready for good associations with partners by and large, and hence builds the work relations and furthermore the air for development in social angles like natural security (Bebchuk et al., 2009). In this way, the examination attempted to review the relationship among execution of the

Revised Manuscript Received on August 05, 2019.

Dr.T.Sita Ramaiah, Department of Finance and Accounting, ICFAI Business School, (IBS), (A Constituent of ICFAI Foundation for Higher Education), Hyderabad, Telangana,India

(E-mail: sitaram2k4@ibsindia.org)

Dr.N.Akbar Jan, Department of Human Resources, ICFAI Business School, (IBS), Hyderabad (A Constituent of ICFAI Foundation for Higher Education), Hyderabad, Telangana,India.

(E-mail: akbarjan75@ibsindia.org)

Dr. Radha Mohan Chebolu, Department of Human Resources, ICFAI Business School, (IBS), Hyderabad (A Constituent of ICFAI Foundation for Higher Education), Hyderabad, Telangana,India.

(E-mail: radhamohan@ibsindia.org)

Thangaraja Arumugam, Vellore Institute of Technology, Chennai, Tamilnadu, India. (E-mail: thangaraja.a@vit.ac.in)

A.K. Subramani, Department of Management Studies, St. Peter's College of Engineering and Technology, Avadi, Chennai, Tamilnadu, India. (E-mail: draksubramani@gmail.com) firm and corporate administration with regards to developing countries are essential to improve appreciation of the subject. In the previous couple of looks into has been embraced to examine the impact of corporate administration on execution of the firm (Chakrabarthi et al., 2007; Desai and Dharmapala, 2011) and there are uncommon research with respect to Indian setting which investigated endogenous between connections among corporate administration, firm execution, proprietorship and capital structure. From now on, the present research centers around impact of corporate administration on firm execution out of sight of creating nation. The focal point of the exploration is to research the relationship among corporate administration and firm execution of S\&P BSE-100 filed organizations and the investigation additionally investigates the between relationship among corporate administration, firm execution, possession, and capital structure.

\section{AIM OF THE STUDY}

The purpose of the examination is to audit the causal relationship among corporate organization and firm execution for the associations enrolled in Bombay Stock Exchange (S\&P BSE-100) and besides to take a gander at the between relationship among corporate organization, capital structure, ownership and execution of the firm.

\section{METHODOLOGY}

\subsection{Data Description}

The examination looks at and surveys the relationship among different factors related to corporate management and implementation of the firm at S\&P BSE-100 enrolled firms. The information for the examination is accomplished. The board informational collection involves 752 perceptions which spreads time arrangement information from 2011-18 and cross segment units of 94 examples firms of the considerable number of factors. Where the required samples is allocated into two major categories of industry such as manufacturing and service and are grouped in to four subindustry wise as information technology, financial services, pharma and manufacturing firms, in order find out differences of corporate governance characteristics industrywise and promote the analysis with findings

Published By:

Blue Eyes Intelligence Engineering 


\subsection{Specification of the Model}

The exploration has utilized pooled relapse models to think about relationship among factors of corporate administration trademark and firm execution. The least squares board information relapse model is utilized both firm and time fakers for first-round to explore the relationship among corporate management and implementation in the firm.

PERF it $=\infty 0+\beta 1$ Blit $+\beta 2$ BSit $+\beta 3$ BRrit $+\beta 4$ PHit + $\beta 5 \mathrm{LEV}$ it

\section{$\gamma^{\mathrm{X}} \mathrm{it}+\mathrm{tt}+\mathrm{di}+\varepsilon i t$}

Correspondingly, though ' $\mathrm{di}^{\prime}$ is signifies firm-explicit impact, while 'tt' speaks with the impact of time and '€it' is the mistake term. The vector of control factors is appeared as 'Xit'. The depiction license for a firm precise fixed impact di, time exertion which is regular to firm gotten by year fakers (tt) and where $€$ it is the arbitrary surreptitiously segment. The pointers of corporate administration are illustrative factors, for example, size of the board, compensation payed to the chiefs, autonomy in the board and capital structure. Past audits demonstrates that the interrelationships between corporate administration, including capital, structure of proprietorship and corporate execution, prescribe that, concentrating the relationship among corporate management and implementation in the firm from an econometric viewpoint needs articulating an arrangement of concurrent conditions that expresses the relationship between the factors (Love, 2010).

\subsection{Variables Measurement}

It is followed in various different mechanisms, which helps the administration to manage a business feasibly which provides advantage for the stakeholders (Morck, 2007). Whereas the entire mechanism of corporate governance is separated into two groups: external and internal. The elements of external mechanism consist of legal framework, market influence, safeguarding the rights of minority ownership. Internal mechanisms consist of size of the board, director remuneration, independence of the board, ownership structure, diversity of board, correlation with stakeholders, lucidity in the present financial processes, reporting and financial leverage (Lipton and Lorsch, 1992). Both external and internal mechanisms are fundamental for responsible the index for computing the corporate governance's quality and have a correlation with firm performance. The corporate governance external mechanism is utilized in studies cross-country. Therefore, the research makes use of corporate governance's internal mechanisms as a proxy for practices of corporate governance.

Table 1 Concepts and measurement of variables

\begin{tabular}{|c|c|c|}
\hline Variables & Abbreviation & Measurement \\
\hline \multicolumn{3}{|l|}{ Dependent variables } \\
\hline Market to Book value & MB & $\begin{array}{l}\text { The ratio of market capitalization of equity to equity of } \\
\text { book value }\end{array}$ \\
\hline Tobin Q & Tobin Q & $\begin{array}{l}\text { (Total assets }+ \text { market value of equity -book value of equity } \\
\text {-deferred taxes)/total assets }\end{array}$ \\
\hline \multicolumn{3}{|l|}{ Independent Variables } \\
\hline Independence of Board & $\mathrm{BI}$ & $\%$ of independent board of directors \\
\hline Size of the Board & BS & Whole number of board of directors \\
\hline Board Remuneration & $\mathrm{Br}$ & $\begin{array}{l}\text { Natural logarithm of total amount payment paid to directors } \\
\text { of the board }\end{array}$ \\
\hline Promoter shareholding & $\mathrm{Ph}$ & $\%$ of equity share ownership / promoter shareholders \\
\hline \multicolumn{3}{|l|}{ Endogenous variables } \\
\hline Return on asset & ROA & Ratio of earning before tax and interest to total assets \\
\hline Leverage & LEV & Ratio of total debt dividend / equity \\
\hline Ownership structure & OS & $\begin{array}{l}\text { Ratio of shares held by director dividend by total } \\
\text { outstanding shares }\end{array}$ \\
\hline Corporate Governance & Gov & Computed index of Corporate governance \\
\hline \multicolumn{3}{|l|}{ Exogenous variables } \\
\hline Size of the firm & LN(TA) & Natural logarithm of total assets of the firm \\
\hline Age of the firm & Age & Number of years of operation \\
\hline Operating performance & EBIT/TA & Operating profit / total assets \\
\hline Dummy Industry & IDUM & $\begin{array}{l}\text { Here ' } 1 \text { ' is used for service and ' } 0 \text { ' is used for } \\
\text { manufacturing with regards to dummy industry. }\end{array}$ \\
\hline
\end{tabular}




\section{RESULTS AND DISCUSSION}

The consequences of the model are given in the tables signify the relapse models result in which Tobin's Q is a reliant variable used to gauge the budgetary presentation of the firm. Model 1 contain perception of all S\&P BSE-100 organizations, Model-2 and Model-3 are set up on perceptions of administrations and assembling segment while Model-4, 5, 6 and 7 are made on its perceptions, Financial, Pharma and other assembling businesses correspondingly.

Table 2 Descriptive Statistics

\begin{tabular}{|c|c|c|c|c|c|c|}
\hline & TQ & BI & BR & BS & PH & LEV \\
\hline \multicolumn{7}{|l|}{ Model-1 } \\
\hline Mean & 3.470137 & 0.449983 & 184.4603 & 14.05615 & 49.00079 & 0.111426 \\
\hline Median & 2.325700 & 0.461540 & 112.9950 & 14.00000 & 52.07375 & 0.028890 \\
\hline Maximum & 39.10210 & 0.812500 & 2970.110 & 29.00000 & 90.00000 & 0.733780 \\
\hline Minimum & 0.094500 & 0.000000 & 0.000000 & 6.000000 & 0.000000 & 0.000000 \\
\hline Std. Dev. & 3.578039 & 0.126918 & 258.1763 & 3.318368 & 19.17241 & 0.168098 \\
\hline \multicolumn{7}{|l|}{ Model-2 } \\
\hline Mean & 4.107993 & 0.454777 & 200.5025 & 14.14935 & 50.79123 & 0.079632 \\
\hline Median & 2.813100 & 0.461540 & 126.7350 & 14.00000 & 52.14500 & 0.038580 \\
\hline Maximum & 39.10210 & 0.812500 & 1913.910 & 29.00000 & 90.00000 & 0.518050 \\
\hline Minimum & 0.337100 & 0.150000 & 0.000000 & 6.000000 & 0.000000 & 0.000000 \\
\hline Std. Dev. & 3.892560 & 0.109060 & 242.3660 & 3.504164 & 16.64373 & 0.100534 \\
\hline \multicolumn{7}{|l|}{ Model-3 } \\
\hline Mean & 2.352044 & 0.442044 & 159.6124 & 13.91259 & 46.36930 & 0.171885 \\
\hline Median & 1.449100 & 0.461540 & 87.22000 & 14.00000 & 51.59375 & 0.007465 \\
\hline Maximum & 28.41310 & 0.785710 & 2970.110 & 26.00000 & 89.78000 & 0.733780 \\
\hline Minimum & 0.094500 & 0.000000 & 1.150000 & 7.000000 & 0.000000 & 0.000000 \\
\hline Std. Dev. & 2.472823 & 0.151326 & 280.8566 & 2.990520 & 22.32201 & 0.232995 \\
\hline \multicolumn{7}{|l|}{\begin{tabular}{|l|} 
Model-4 \\
\end{tabular}} \\
\hline Mean & 1.855585 & 0.408219 & 65.97511 & 13.50350 & 37.28528 & 0.176247 \\
\hline Median & 1.190200 & 0.454550 & 31.45000 & 13.00000 & 40.31000 & 0.000000 \\
\hline Maximum & 28.41310 & 0.777780 & 307.8300 & 24.00000 & 89.78000 & 0.733780 \\
\hline Minimum & 0.911400 & 0.000000 & 1.150000 & 8.000000 & 0.000000 & 0.000000 \\
\hline Std. Dev. & 2.857401 & 0.188470 & 73.24931 & 3.053223 & 22.59093 & 0.260601 \\
\hline \multicolumn{7}{|l|}{ Model-5 } \\
\hline Mean & 3.847400 & 0.526839 & 435.7555 & 14.35000 & 55.00981 & 0.016160 \\
\hline Median & 3.606100 & 0.500000 & 247.4150 & 14.00000 & 63.47375 & 0.001410 \\
\hline Maximum & 8.944200 & 0.785710 & 2970.110 & 20.00000 & 79.41000 & 0.105030 \\
\hline Minimum & 0.405300 & 0.375000 & 21.63000 & 9.000000 & 12.75000 & 0.000000 \\
\hline Std. Dev. & 2.074085 & 0.107843 & 615.1405 & 2.413105 & 23.60921 & 0.027536 \\
\hline \multicolumn{7}{|l|}{\begin{tabular}{|l|} 
Model-6 \\
\end{tabular}} \\
\hline Mean & 4.580539 & 0.477455 & 284.0863 & 11.58333 & 51.94368 & 0.034987 \\
\hline Median & 3.941050 & 0.458045 & 226.0000 & 12.00000 & 52.08000 & 0.003420 \\
\hline Maximum & 13.10480 & 0.727270 & 1079.260 & 16.00000 & 74.79000 & 0.158230 \\
\hline Minimum & 0.726700 & 0.166670 & 35.21000 & 7.000000 & 25.49750 & 0.000000 \\
\hline Std. Dev. & 2.428330 & 0.101751 & 235.2076 & 1.962680 & 14.37575 & 0.048338 \\
\hline \multicolumn{7}{|l|}{ Model-7 } \\
\hline Mean & 4.021198 & 0.450590 & 185.0716 & 14.62308 & 50.57955 & 0.087832 \\
\hline Median & 2.496200 & 0.461540 & 109.4300 & 15.00000 & 52.35250 & 0.050305 \\
\hline Maximum & 39.10210 & 0.812500 & 1913.910 & 29.00000 & 90.00000 & 0.518050 \\
\hline Minimum & 0.337100 & 0.150000 & 0.000000 & 6.000000 & 0.000000 & 0.000000 \\
\hline Std. Dev. & 4.101551 & 0.109968 & 240.7996 & 3.522091 & 17.03548 & 0.105402 \\
\hline
\end{tabular}

NOTE: TQ - Tobin's Q, BI - Board Independence, BR - Board Remuneration, BS - Board Size, LEV - Leverage , PH Promoter Shareholding. 
In the Model 1, the average of the board size ranges from 6 to 29 members with the mean value of 14.05 (median = 14.00) and standard deviation of 3.31. The board independence ranges from 0.00 to 0.81 with the mean value of 0.44 (median $=0.46$ ) and standard deviation 0.12 . Similarly, with regard to board remuneration, the range varies from 0.00 to 2970.11 with the mean value of 184.46 (median $=112.99)$ and standard deviation 258.16. The Tobin's Q value also varies from 0.33 to 39.10 with the mean value of 4.10 (median $=2.81$ ) and standard deviation 3.89. The above analysis of Model 1 reveals a normal distribution and no major skewness can be detected. . Similarly, in all remaining models also, we did not find major skewness.

Table 3 Output of panel data Model - Pooled regression

\begin{tabular}{|c|c|c|c|c|c|c|c|c|c|c|c|c|c|c|}
\hline & \multicolumn{2}{|c|}{ Model 1} & \multicolumn{2}{|c|}{ Model 2} & \multicolumn{2}{|c|}{ Model 3} & \multicolumn{2}{|c|}{ Model 4} & \multicolumn{2}{|c|}{ Model 5} & \multicolumn{2}{|c|}{ Model 6} & \multicolumn{2}{|c|}{ Model 7} \\
\hline & $\begin{array}{l}\stackrel{\overrightarrow{0}}{0} \\
\stackrel{0}{0} \\
0 \\
0 \\
0\end{array}$ & 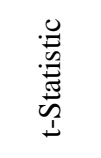 & 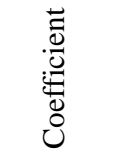 & 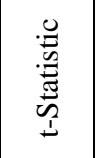 & 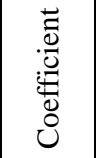 & 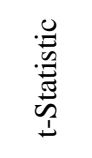 & 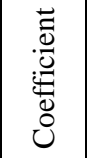 & 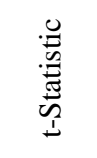 & 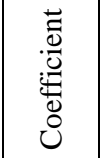 & 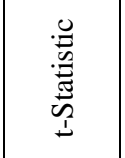 & 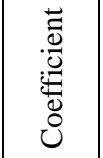 & 苞 & 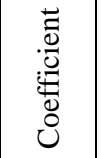 & 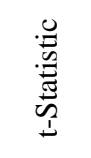 \\
\hline $\mathrm{C}$ & 5.259 & $\begin{array}{c}5.731 \\
* * *\end{array}$ & 7.372 & $\begin{array}{c}5.426 \\
* * *\end{array}$ & 6.171 & $\begin{array}{l}2.960 \\
* * *\end{array}$ & 6.171 & 1.806 & 13.131 & $\begin{array}{c}2.888 \\
* * *\end{array}$ & -1.198 & -0.371 & 8.415 & $\begin{array}{c}5.568 \\
* * *\end{array}$ \\
\hline BI & 1.316 & 1.269 & 0.716 & 0.448 & 1.691 & 1.644 & 0.985 & 0.576 & -6.942 & $-2.033 * *$ & 2.664 & 0.994 & 0.660 & 0.365 \\
\hline $\mathrm{BR}$ & 0.000 & 0.472 & 0.000 & -0.273 & 0.000 & 0.751 & 0.007 & 1.886 & -0.002 & $-2.330 * *$ & -0.001 & -1.091 & 0.000 & 0.264 \\
\hline $\mathrm{BS}$ & -0.226 & $\begin{array}{c}-5.922 \\
* * *\end{array}$ & -0.248 & $\begin{array}{c}-4.935 \\
* * *\end{array}$ & -0.148 & $\begin{array}{c}-3.035 \\
* * *\end{array}$ & -0.265 & $\begin{array}{c}-2.919 \\
* * *\end{array}$ & -0.323 & -1.786 & 0.118 & 0.773 & -0.300 & $\begin{array}{c}-5.259 \\
* * *\end{array}$ \\
\hline LEV & -5.984 & $\begin{array}{c}-8.240 \\
* * *\end{array}$ & -10.518 & $\begin{array}{c}-6.171 \\
* * *\end{array}$ & -3.225 & $\begin{array}{c}-5.242 \\
* * *\end{array}$ & -3.093 & $\begin{array}{c}-3.262 \\
* * *\end{array}$ & 29.488 & $-2.458 * *$ & $\begin{array}{c}- \\
19.476\end{array}$ & $\begin{array}{c}-3.310 \\
* * *\end{array}$ & 10.666 & $\begin{array}{c}-5.759 \\
* * *\end{array}$ \\
\hline $\mathrm{PH}$ & 0.029 & $\begin{array}{c}4.468 \\
* * *\end{array}$ & 0.016 & 1.440 & 0.025 & $\begin{array}{c}3.959 \\
* * *\end{array}$ & 0.044 & $\begin{array}{c}3.255 \\
* * *\end{array}$ & 0.003 & 0.142 & 0.080 & $\begin{array}{c}4.001 \\
* * *\end{array}$ & 0.011 & 0.931 \\
\hline $\begin{array}{c}\text { Adjusted } \\
\text { R-squared }\end{array}$ & \multicolumn{2}{|c|}{0.147} & \multicolumn{2}{|c|}{0.154} & \multicolumn{2}{|c|}{0.134} & \multicolumn{2}{|c|}{0.135} & \multicolumn{2}{|c|}{0.169} & \multicolumn{2}{|c|}{0.236} & \multicolumn{2}{|c|}{0.162} \\
\hline F-statistic & \multicolumn{2}{|c|}{26.50311} & \multicolumn{2}{|c|}{17.75083} & \multicolumn{2}{|c|}{9.713058} & \multicolumn{2}{|c|}{5.309114} & \multicolumn{2}{|c|}{2.582362} & \multicolumn{2}{|c|}{5.391567} & \multicolumn{2}{|c|}{16.01005} \\
\hline Observation & \multicolumn{2}{|c|}{752} & \multicolumn{2}{|c|}{464} & \multicolumn{2}{|c|}{288} & \multicolumn{2}{|c|}{144} & \multicolumn{2}{|c|}{40} & \multicolumn{2}{|c|}{72} & \multicolumn{2}{|c|}{392} \\
\hline
\end{tabular}

The above table gives the investigation of the relationship among Tobin's $\mathrm{Q}$ and board freedom (BI), board compensation (BR), board size (BS), influence (LEV) and advertiser shareholding $(\mathrm{PH})$. On account of Model 1, the board size (- 0.226) and influence (- 5.984) affects Tobin's Q while the advertiser shareholding (0.029) positively affects Tobin's Q. Likewise, on account of Model 2 just the board size (- 0.248) and influence (- 10.518) affects Tobin's Q. On account of Model 3 the board size (- 0.148) and influence (3.225) affects Tobin's $Q$ while the advertiser shareholding (0.025) positively affects Tobin's Q. With respects Model 4 the board size $(-0.265)$ and influence (- 3.093) negatively affects Tobin's Q while the advertiser shareholding (0.044) impacts Tobin's Q. On account of Model 5 the board autonomy (- 6.942), board compensation (- 0.002) and influence (- 29.488) negatively affects Tobin's Q. On account of Model 6 the influence (-19.476) and advertiser shareholding (0.080) negatively affects Tobin's Q. On account of Model 7 the board size (-0.300) and influence (10.666) impacts Tobin's Q.

The pooled relapse yield gave the model portrayal of corporate administration variables and its effect on the Tobin's Q. To decide and pick the board impact of information the specialist has utilized Hausman test. The theories fixed for board examination are as per the following:

Ho - Random impact relapse model is reasonable.

H1 - Fixed impact relapse model is reasonable. 
Table 4 Hausman Test Panel 1

\begin{tabular}{|l|l|l|l|l|}
\hline Model & $X^{2}$ Statistic & $X^{2}$ d.f. & Prob. & Appropriate test \\
\hline Model 1 & 10.52849 & 5 & 0.0616 & Random effect model \\
\hline Model 2 & 6.573032 & 5 & 0.2544 & Random effect model \\
\hline Model 3 & 1.963505 & 5 & 0.8542 & Random effect model \\
\hline Model 4 & 4.757356 & 5 & 0.4462 & Random effect model \\
\hline Model 5 & 18.61938 & 5 & 0.0023 & Fixed effect model \\
\hline Model 6 & 8.65443 & 5 & 0.1237 & Random effect model \\
\hline Model 7 & 4.128453 & 5 & 0.5311 & Random effect model \\
\hline
\end{tabular}

The above table shows when to use the fixed effects panel data regression analysis and when to use random effects panel data regression analysis. If the $\mathrm{Chi}-\mathrm{Sq}$ probability value is less than 0.05 then fixed effects panel data regression is appropriate and if the Chi-Sq probability value is more than 0.05 then random effects panel data regression should be used. Only in the case of Model 5 the probability value is less than $0.05(0.0023)$ and therefore it is necessary to do fixed effect panel data analysis. In the case of other models, since the probability value is more than 0.05 , it is required to do random effect panel data analysis.

Table 5 Output of panel data Model -Random effect: Association among firm performance dignified by Tobin Q's and corporate governance

\begin{tabular}{|c|c|c|c|c|c|c|c|c|c|c|c|c|c|c|}
\hline & \multicolumn{2}{|c|}{ Model 1} & \multicolumn{2}{|c|}{ Model 2} & \multicolumn{2}{|c|}{ Model 3} & \multicolumn{2}{|c|}{ Model 4} & \multicolumn{2}{|c|}{ Model 5} & \multicolumn{2}{|c|}{ Model 6} & \multicolumn{2}{|c|}{ Model 7} \\
\hline & \begin{tabular}{l}
\multirow{4}{0}{} \\
$\vdots$ \\
0 \\
0
\end{tabular} & $\frac{\varrho}{ٍ}$ & \begin{tabular}{l}
\multirow{4}{0}{} \\
$\vdots$ \\
0
\end{tabular} & $\frac{0}{\stackrel{0}{\pi}}$ & \begin{tabular}{l}
\multirow{4}{0}{} \\
$\dot{1}$ \\
0
\end{tabular} & 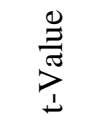 & \begin{tabular}{l}
\multirow{4}{0}{} \\
$\vdots$ \\
0 \\
0
\end{tabular} & 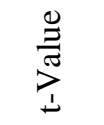 & 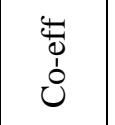 & 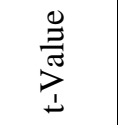 & \begin{tabular}{l}
\multirow{4}{0}{} \\
$\vdots$ \\
0 \\
0
\end{tabular} & 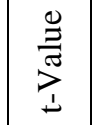 & 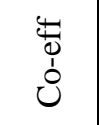 & 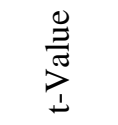 \\
\hline $\mathrm{C}$ & 7.997 & $2.266 * *$ & 7.416 & $\begin{array}{c}5.466 \\
* * *\end{array}$ & 2.908 & $\begin{array}{c}2.827 \\
* * *\end{array}$ & -0.132 & -0.046 & 2.550 & 1.592 & 8.623 & 0.816 & 8.490 & $\begin{array}{c}5.602 \\
* * *\end{array}$ \\
\hline BI & -3.937 & -1.513 & 0.697 & 0.437 & 1.948 & 1.844 & -0.262 & -0.126 & -0.057 & -0.048 & 0.730 & 0.239 & 0.617 & 0.341 \\
\hline BR & -0.001 & -1.262 & 0.000 & -0.316 & 0.000 & 0.348 & 0.004 & 1.045 & 0.000 & 0.335 & -0.001 & $-0.67 \mid$ & 0.000 & 0.198 \\
\hline BS & -0.133 & -0.942 & -0.250 & $\begin{array}{c}-4.988 \\
* * *\end{array}$ & -0.149 & $\begin{array}{c}-3.033 \\
* * *\end{array}$ & 0.044 & 0.399 & 0.060 & 1.082 & -0.273 & -1.21 & -0.302 & $\begin{array}{c}-5.319 \\
* * *\end{array}$ \\
\hline LEV & -65.554 & $\begin{array}{c}-5.946 \\
* * *\end{array}$ & $\begin{array}{c}- \\
10.507 \\
\end{array}$ & $\begin{array}{c}-6.190 \\
* * *\end{array}$ & -3.280 & $\begin{array}{c}-5.293 \\
* * * \\
\end{array}$ & 2.250 & 0.276 & 0.006 & 0.210 & -7.725 & -0.99 & -10.65 & $\begin{array}{c}-5.763 \\
* * *\end{array}$ \\
\hline $\mathrm{PH}$ & 0.022 & 1.584 & 0.016 & 1.436 & 0.026 & $\begin{array}{c}4.058 \\
* * *\end{array}$ & 0.023 & 0.426 & -1.809 & -0.774 & -0.012 & $-0.07 d$ & 0.011 & 0.920 \\
\hline $\begin{array}{c}\text { Adjusted R- } \\
\text { squared }\end{array}$ & \multicolumn{2}{|c|}{0.576} & \multicolumn{2}{|c|}{0.155} & \multicolumn{2}{|c|}{0.126} & \multicolumn{2}{|c|}{0.502} & \multicolumn{2}{|c|}{0.523} & \multicolumn{2}{|c|}{0.292} & \multicolumn{2}{|c|}{0.164} \\
\hline F-Value & \multicolumn{2}{|c|}{6.350934} & \multicolumn{2}{|c|}{17.91953} & \multicolumn{2}{|c|}{4.389213} & \multicolumn{2}{|c|}{7.315224} & \multicolumn{2}{|c|}{9.285925} & \multicolumn{2}{|c|}{3.253903} & \multicolumn{2}{|c|}{16.23783} \\
\hline $\begin{array}{c}\text { Hausman } \\
\text { Test } \\
\text { (Prob.) } \\
\end{array}$ & \multicolumn{2}{|c|}{0.062} & \multicolumn{2}{|c|}{0.254} & \multicolumn{2}{|c|}{0.854} & \multicolumn{2}{|c|}{0.446} & \multicolumn{2}{|c|}{0.002} & \multicolumn{2}{|c|}{0.051} & \multicolumn{2}{|c|}{0.531} \\
\hline Total & \multicolumn{2}{|c|}{752} & \multicolumn{2}{|c|}{464} & \multicolumn{2}{|c|}{288} & \multicolumn{2}{|c|}{144} & \multicolumn{2}{|c|}{40} & \multicolumn{2}{|c|}{72} & \multicolumn{2}{|c|}{392} \\
\hline
\end{tabular}

The above table gives the examination of the relationship among Tobin's Q and board autonomy (BI), board compensation (BR), board size (BS), influence (LEV) and advertiser shareholding $(\mathrm{PH})$. On account of Model 1, the influence (- 65.554) has a negative impact on Tobin's Q. On account of Model 2, just the board size (- 0.250) and influence (- 10.507) impacts Tobin's Q. On account of Model 3 the board size (-0.149) and influence (-3.280) has a negative impact on Tobin's $Q$ while the advertiser shareholding (0.026) positively affects Tobin's Q. On account of Model 4 there is no effect on Tobin's Q. On account of Model 5 there is no impact on Tobin's Q. On account of Model 6 there is no effect on Tobin's Q. With deference Model 7 the board size (- 0.302) and influence (10.650) affects Tobin's Q. It is seen from every one of the models, that the time and firm impacts are significant speaking to presence of varieties in segment and industrywise on factors of corporate administration and execution of the firm. 
Table 6 Descriptive Statistics

\begin{tabular}{|c|c|c|c|c|c|c|}
\hline & MB & BI & BR & BS & PH & LEV \\
\hline \multicolumn{7}{|l|}{ Model-1 } \\
\hline Mean & 5.376112 & 0.449983 & 184.4603 & 14.05615 & 49.00079 & 0.111426 \\
\hline Median & 3.419200 & 0.461540 & 112.9950 & 14.00000 & 52.07375 & 0.028890 \\
\hline Maximum & 44.10930 & 0.812500 & 2970.110 & 29.00000 & 90.00000 & 0.733780 \\
\hline Minimum & 0.337900 & 0.000000 & 0.000000 & 6.000000 & 0.000000 & 0.000000 \\
\hline Std. Dev. & 6.280278 & 0.126918 & 258.1763 & 3.318368 & 19.17241 & 0.168098 \\
\hline \multicolumn{7}{|l|}{ Model-2 } \\
\hline Mean & 6.648481 & 0.456236 & 203.7700 & 14.08874 & 51.55997 & 0.069290 \\
\hline Median & 4.055450 & 0.461540 & 134.2750 & 14.00000 & 52.79750 & 0.029460 \\
\hline Maximum & 44.10930 & 0.812500 & 1913.910 & 29.00000 & 90.00000 & 0.389670 \\
\hline Minimum & 0.337900 & 0.150000 & 0.000000 & 6.000000 & 0.000000 & 0.000000 \\
\hline Std. Dev. & 7.450949 & 0.109908 & 243.4323 & 3.507464 & 16.75913 & 0.088566 \\
\hline \multicolumn{7}{|l|}{ Model-3 } \\
\hline Mean & 3.677151 & 0.442706 & 155.9110 & 13.95455 & 46.30512 & 0.169110 \\
\hline Median & 2.855000 & 0.457275 & 79.05000 & 14.00000 & 52.03750 & 0.001320 \\
\hline Maximum & 28.94760 & 0.785710 & 2970.110 & 26.00000 & 89.78000 & 0.733780 \\
\hline Minimum & 0.584300 & 0.000000 & 1.150000 & 7.000000 & 0.000000 & 0.000000 \\
\hline Std. Dev. & 2.960388 & 0.153147 & 280.9595 & 2.999654 & 22.39851 & 0.234073 \\
\hline \multicolumn{7}{|l|}{ Model-4 } \\
\hline Mean & 2.962790 & 0.408219 & 65.97511 & 13.50350 & 37.28528 & 0.176247 \\
\hline Median & 2.266350 & 0.454550 & 31.45000 & 13.00000 & 40.31000 & 0.000000 \\
\hline Maximum & 28.94760 & 0.777780 & 307.8300 & 24.00000 & 89.78000 & 0.733780 \\
\hline Minimum & 0.584300 & 0.000000 & 1.150000 & 8.000000 & 0.000000 & 0.000000 \\
\hline Std. Dev. & 2.995700 & 0.188470 & 73.24931 & 3.053223 & 22.59093 & 0.260601 \\
\hline \multicolumn{7}{|l|}{ Model-5 } \\
\hline Mean & 5.119300 & 0.526839 & 435.7555 & 14.35000 & 55.00981 & 0.016160 \\
\hline Median & 4.596850 & 0.500000 & 247.4150 & 14.00000 & 63.47375 & 0.001410 \\
\hline Maximum & 11.64620 & 0.785710 & 2970.110 & 20.00000 & 79.41000 & 0.105030 \\
\hline Minimum & 2.118500 & 0.375000 & 21.63000 & 9.000000 & 12.75000 & 0.000000 \\
\hline Std. Dev. & 2.282423 & 0.107843 & 615.1405 & 2.413105 & 23.60921 & 0.027536 \\
\hline \multicolumn{7}{|l|}{ Model-6 } \\
\hline Mean & 6.300092 & 0.477455 & 284.0863 & 11.58333 & 51.94368 & 0.034987 \\
\hline Median & 5.271600 & 0.458045 & 226.0000 & 12.00000 & 52.08000 & 0.003420 \\
\hline Maximum & 17.55990 & 0.727270 & 1079.260 & 16.00000 & 74.79000 & 0.158230 \\
\hline Minimum & 0.994300 & 0.166670 & 35.21000 & 7.000000 & 25.49750 & 0.000000 \\
\hline Std. Dev. & 3.761890 & 0.101751 & 235.2076 & 1.962680 & 14.37575 & 0.048338 \\
\hline \multicolumn{7}{|l|}{ Model-7 } \\
\hline Mean & 6.712471 & 0.452319 & 188.9423 & 14.55128 & 51.48949 & 0.075591 \\
\hline Median & 3.528850 & 0.461540 & 116.2300 & 14.00000 & 52.97750 & 0.037690 \\
\hline Maximum & 44.10930 & 0.812500 & 1913.910 & 29.00000 & 90.00000 & 0.389670 \\
\hline Minimum & 0.337900 & 0.150000 & 0.000000 & 6.000000 & 0.000000 & 0.000000 \\
\hline Std. Dev. & 7.946284 & 0.111028 & 242.3133 & 3.535525 & 17.17642 & 0.092778 \\
\hline
\end{tabular}

The above table provides the descriptive analysis of the variables

Model 1: The normal of the board size in Model 1 territories from 6 to 29 individuals with the mean estimation of 14.05 (middle $=14.00$ ) and standard deviation of 3.31 . The board freedom ranges from 0.00 to 0.81 with the mean estimation of 0.44 (middle $=0.46$ ) and standard deviation 0.12 . Also, concerning board compensation, the range shifts from 0.00 to 2970.11 with the mean estimation of 184.46 (middle $=112.99)$ and standard deviation 258.16. The MB esteem likewise fluctuates from 0.33 to 44.10 with the mean estimation of 5.37 (middle $=3.41$ ) and standard deviation 6.28. The above investigation of Model 1 uncovers a typical dissemination and no real skewness can be recognized. Correspondingly, in every single staying model additionally, we didn't discover significant skewness.

\section{SLS Model output:}

The 2SLS condition models employed for assessment of endogenous connection among performance of firm and corporate governance. The Hausman test is undertaken on every framework to figure out which valuation strategy is normally suitable.

Structural Equation Estimates: 
$\mathrm{ROA}=\alpha+\beta 1 \mathrm{OS}+\beta 2 \mathrm{GOV}+\beta 3 \mathrm{LEV}+\beta 4 \mathrm{BS}+\beta 5 \mathrm{LN}$ (TA) $+\beta 6 \mathrm{BA}+\epsilon 1$

$\mathrm{GOV}=\alpha+\beta 1 \mathrm{OS}+\beta 2 \mathrm{ROA}+\beta 3 \mathrm{LEV}+\beta 4 \mathrm{BS}+\beta 6 \mathrm{BI}+$ $\beta 6 \mathrm{BR}+\epsilon 2$
$\mathrm{OS}=\alpha+\beta 1 \mathrm{ROA}+\beta 2 \mathrm{GOV}+\beta 3 \mathrm{LEV}+\beta 4 \mathrm{BS}+\beta 5 \mathrm{LN}$ (TA) $+\beta 6 \mathrm{EBIT}+\epsilon 3$

$\mathrm{LEV}=\alpha+\beta 1 \mathrm{OS}+\beta 2 \mathrm{GOV}+\beta 3 \mathrm{ROA}+\beta 4 \mathrm{BS}+\beta 5 \mathrm{BA}$

$+\beta 6 \mathrm{IDUM}+\epsilon 4$

Table 11 Output of Two-stage Least Squares (2SLS) Model

\begin{tabular}{|c|c|c|c|c|c|c|c|c|}
\hline & \multicolumn{2}{|l|}{ MODEL A } & \multicolumn{2}{|l|}{ MODEL B } & \multicolumn{2}{|c|}{ MODEL C } & \multicolumn{2}{|l|}{ MODEL D } \\
\hline & Coefficient & $\begin{array}{l}\text { Std. } \\
\text { Error }\end{array}$ & Coefficient & $\begin{array}{l}\text { Std. } \\
\text { Error }\end{array}$ & $\begin{array}{l}\text { Coeffici } \\
\text { ent }\end{array}$ & Std. Error & Coefficient & Std. Error \\
\hline constant & -0.201395 & 0.156753 & $0.412589 * *$ & 0.054698 & $\begin{array}{l}60.3608 \\
7 * *\end{array}$ & 3.148756 & $\begin{array}{l}0.101981 * \\
*\end{array}$ & 0.041487 \\
\hline ROA & & & $0.046452 * *$ & 0.013853 & $\begin{array}{l}4.85675 \\
4 * *\end{array}$ & 0.929753 & $\begin{array}{l}- \\
0.039225 * \\
*\end{array}$ & 0.009941 \\
\hline GOV & $0.305147 * *$ & 0.092909 & & & $\begin{array}{l}1.46752 \\
9\end{array}$ & 2.255753 & -0.014431 & 0.023577 \\
\hline OS & $0.009783 * *$ & 0.001482 & 0.000360 & 0.000605 & & & $\begin{array}{l}0.000767 * \\
*\end{array}$ & 0.000385 \\
\hline LEV & $\begin{array}{l}- \\
1.019123 * *\end{array}$ & 0.139501 & -0.053662 & 0.056828 & $\begin{array}{l}- \\
0.17135 \\
0 * *\end{array}$ & 0.177805 & & \\
\hline BS & $0.022332 * *$ & 0.007409 & & & & & $\begin{array}{l}0.000264 * \\
*\end{array}$ & 0.001815 \\
\hline $\mathrm{BA}$ & $0.002703 * *$ & 0.000951 & & & & & $\begin{array}{l}- \\
0.000638 * \\
*\end{array}$ & 0.000240 \\
\hline LN(TA) & $\begin{array}{l}-7.35 \mathrm{E}- \\
08 * *\end{array}$ & 9.34E-09 & & & $\begin{array}{l}-3.60 \mathrm{E}- \\
07\end{array}$ & $2.35 \mathrm{E}-07$ & & \\
\hline BI & & & 0.144063 & 0.075617 & & & & \\
\hline BR & & & $\begin{array}{l}-9.66 \mathrm{E}- \\
05 * *\end{array}$ & $3.63 \mathrm{E}-05$ & & & & \\
\hline EBIT & & & & & $\begin{array}{l}13.1287 \\
3 \\
\end{array}$ & 7.030667 & & \\
\hline IDUM & & & & & & & $\begin{array}{l}0.062232 * \\
*\end{array}$ & 0.014367 \\
\hline R-squared & \multicolumn{2}{|l|}{0.218269} & \multicolumn{2}{|l|}{0.034827} & \multicolumn{2}{|l|}{0.080947} & \multicolumn{2}{|l|}{0.107063} \\
\hline $\begin{array}{l}\text { Adjusted R- } \\
\text { squared }\end{array}$ & \multicolumn{2}{|l|}{0.211939} & \multicolumn{2}{|l|}{0.028279} & \multicolumn{2}{|l|}{0.073505} & \multicolumn{2}{|l|}{0.099833} \\
\hline F statistics & \multicolumn{2}{|l|}{$34.48269^{*}$} & \multicolumn{2}{|l|}{$5.318715^{*}$} & \multicolumn{2}{|l|}{$10.87740 *$} & \multicolumn{2}{|l|}{$14.80763 *$} \\
\hline \multicolumn{9}{|c|}{ Hausman Specification Test (OLS Vs. 2SLS): } \\
\hline h-Stat & \multicolumn{2}{|c|}{$91.943 *$} & \multicolumn{2}{|l|}{$93.292 *$} & \multicolumn{2}{|c|}{$87.301 *$} & \multicolumn{2}{|l|}{$81.066^{*}$} \\
\hline \multicolumn{9}{|c|}{ Stock and Yogo Weak Instruments Test: } \\
\hline $\begin{array}{l}\text { First-Stage } \\
\text { F-stats (For } \\
\text { Endogenous } \\
\text { variables) }\end{array}$ & \multicolumn{2}{|c|}{$67.23,72.91,59.66$} & \multicolumn{2}{|c|}{$71.93,77.82,68.33$} & \multicolumn{2}{|c|}{$69.23,71.02,70.38$} & \multicolumn{2}{|c|}{$66.39,70.82,67.25$} \\
\hline $\begin{array}{l}\text { Critical } \\
\text { Value }(5 \%)\end{array}$ & \multicolumn{2}{|l|}{19.28} & \multicolumn{2}{|l|}{19.28} & \multicolumn{2}{|c|}{19.28} & \multicolumn{2}{|l|}{19.28} \\
\hline
\end{tabular}

The result of 2SLS model unveils the event of endogeneity in firm execution and administration. The ends show that organizations receiving total system of administration recorded a more beneficial ROA. Consequently administration pursues executed by the firm have an impact of ROA, which relies upon the possession structure and consideration. Thus, the examination distinguishes bidirectional relationship among corporate administration and ROA.

\section{CONCLUSION}

it is set up that influence and bigger board size is impacting execution of the firm (Lipton and Lorsch, 1992). The result of the investigation additionally demonstrates that the recompenses gotten by the immense board are

Published By: 
outperformed moderate basic leadership process, where no trade of thought concerning managerial execution and absence of hazard taking disposition. Little sheets are more plausible than gigantic sheets since they have a high level of coordination and inclusion, with not many challenges looked concerning correspondence, and a lower event of issues of extraordinary free-rider. In addition, executives with autonomous charge are extremely less propelled to work effectively when the size of the sheets get bigger, subsequently it turns out to be progressively dangerous for the free chiefs to impart their insight and contemplations which effect the proficiency of settling on choice. Opportunity of the board individuals in firms Indian is build up to be influence firms execution (Bekiris 2013) where higher freedom to the chief on the board gives dynamically mindful oversight of the observing system. The exploration additionally uncovers that more prominent degree of budgetary motivators to the executives thus lead to more noteworthy level execution of firm (Meckling 1976). It prescribes Indian firms need to actualize best practices of corporate administration for enhancement of both money related and showcase execution. The momentum research has edified on systems of interior corporate administration, anyway the future inquires about may accentuation on the impact of outer administration components, for example, hostile to takeover arrangements, FDI, corporate control, work market and execution.

\section{REFERENCES}

1. Aghion, P., Caroli, E. and Garcia-Penalosa, C. (1999) 'Inequality and economic growth: the perspective of the new growth theories', Journal of Economic Literature, Vol. 37, No. 4, pp.1615-1660.

2. Aggarwal, Reena and Erel, Isil and Stulz, Rene M. and Williamson, Rohan G. (2007) 'Do U.S. Firms Have the Best Corporate Governance? A Cross-Country Examination of the Relation between Corporate Governance and Shareholder Wealth', Fisher College of Business Working Paper No. 2006-03-006; ECGI Finance Working Paper No. 145/2007; Charles A. Dice Center Working Paper No. 2006-25.

3. Anderson, R.C. and Reeb, D.M. (2003). 'FoundingFamily Ownership and Firm Performance: Evidence from the S\&P 500', Journal of Finance, Vol. 58, pp.1301-1328.

4. Balezentis, A. and Balkiene, K. (2014) 'Innovation policy measurement: analysis of Lithuania's case', Economic Research-Ekonomska Istraživanja, Vol. 27, No. 1, 1-14.

5. Bekiris, F.V. (2013). 'Ownership structure and board structure: are corporate governance mechanisms interrelated?', Corporate Governance: The international journal of business in society, Vol. 13, No. 4, pp. 352364.

6. Claessens, S. (2006). Corporate governance and development. The World bank research observer, Vol. 21, No. 1, pp. 91-122.

7. Davis, J.H., Schoorman, F.D., and Donaldson, L. (1997) 'Toward a Stewardship Theory of Management' The Academy of Management Review, Vol. 22, No. (1), pp.20- 47.

8. Davidson, W.N. (2003) 'Agency cost, ownership structure and corporate governance mechanisms', Journal of Banking and Finance, Vol. 27, pp.793-816.
9. Desai, M., and Dharmapala, D., (2011) 'Dividend taxes and international portfolio choice', The Review of Economics and Statistics, Vol. 93, pp.266-284

10. Denis, D.J., and Kruse, T.A. (2000) 'Managerial discipline and corporate restructuring following performance declines', Journal of Financial Economics, Vol. 55, pp.391-424.

11. Hausman, J. (1978). 'Specification tests in Econometrics', Econometrica, Vol. 46, pp. 1251-1271.

12. Hermalin, B.E. and Weisbach, M.S. (2001) 'Boards of directors as an endogenously determined institution: A survey of the economic literature', FRBNY Economic Policy Review, pp.7-26.

13. Jensen, M.C. (1993) 'The modern industrial revolution, exit, and the failure of internal control systems'. Journal of Finance, Vol. 48, pp. 831-880. Jensen, M. C., \& Meckling, W. (1976). Theory of the firm: Managerial behaviour, agency cost and ownership structure. Journal of Financial Economics, Vol. 3, pp.305-360.

14. Jensen, M.C., and Meckling, M.H., (1976) 'Theory of the firm: Managerial behaviour, agency costs and ownership structure', Journal of Financial Economics, Vol. 20, pp.305-360. 